\title{
New Multimedia Platform for Improving Chemotherapy Prescription in Veterinary Patologic Oncology - A Marketing Study
}

\author{
Mihai Marian BORZAN ${ }^{1,4^{*}}$, Nicolae TODOR ${ }^{3}$, Laura Elisabeta CIULEANU, Alexandru Raul POP², Tudor \\ Eliade CIULEANU 3,4 \\ ${ }^{1}$ Department of Animal Productions and Food Safety, USAMV Cluj, Romania \\ ${ }^{2}$ Department of Reproduction, Obstetrics and Reproductive Pathology, USAMV Cluj, Romania \\ ${ }^{3}$ Oncology Institute "Prof. Dr. Ion Chiricuță , \\ ${ }^{4}$ University of Medicine and Pharmacy "Iuliu Hatieganu" Cluj-Napoca \\ Corresponding author: mihai_borzan@yahoo.com
}

Bulletin UASVM Veterinary Medicine 72(1) / 2015,

Print ISSN 1843-5270; Electronic ISSN 1843-5378

DOI:10.15835/buasvmcn-vm: 11088

\begin{abstract}
We conducted a study based on a survey which was original developed in a focus group with veterinarians to assess the necessity felt and expressed to optimize the chemotherapy prescription in oncological veterinary medicine, the development and implementation of a multimedia platform.

The aim of this research is to test the acceptability regarding the realization and implementation of a multimedia platform to prescribe cytostatic medical treatment in the veterinary medicine.

Participants in the study belonged to both genders in almost equal percentages (F: 50.94\% and M: 49.06\%), the majority were aged between 25-59 years, with activities in the field of veterinary medicine, with experience between 1 and 29 years.

Research has revealed a high degree of utility of the platform, efficiency of information, its positive influence on the quality of care and increased availability of electronic access and purchasing power for the two types of applications - computer (on line) and mobile phone applications.
\end{abstract}

Keywords: cytostatic prescription, marketing software, multimedia platform, veterinary oncology.

\section{INTRODUCTION}

In veterinary medicine practice the cytostatic treatment is available mainly in printed form books, articles, studies, researches. Due to ethical issues regarding the limited access to therapeutic protocols for the pets, through the elevated costs of the chemotherapy and in the absence of the animal health insurance for pets in case of cancer in Romania, in the literature (Reif, 2007) it is accepted the idea to introduce in practice the software for oncological therapy depending on the localization, type, infiltration of tumor, based on standardized therapeutic protocols which are international recognized.
The data available for the incidence of neoplazic tumors in dogs and cats are limited and are not actualized. One of the most important sources of information remains today California Animal Neoplasm Registry (Reif, 2007; Dorn et al., 1968).

The mammary cancer is considered to be the most frequent form of cancer in dogs, with an incidence of 198,88 per 100.000 dogs per year and a relative frequency 7 times higher than in cats and 5 times higher than in humans. Although the mammary gland tumors are present in all species, they are more frequent in bitches (Groza et al., 2006). 
A more recent study (2002), where the assured dogs from UK were subjected, showed a close annual ratio of 205 per 100.000 dogs per year (Dobson et al., 2002). Another research (Egenvall et al., 2005) on 80000 assured bitches, conducted in Sweden, showed an increasing incidence of tumors with age: at 6 years, the risk is $1 \%$; at 8 years is $8 \%$ : and at 10 years is $13 \%$.

The prognostic for this type of disease is reserved; the average survival is 28 months for the small tumors, without regional adenopathology and distal metastasis, and only 5 months in case of distal metastasis at the time of diagnosis (Philibert et al., 2003).

In contrast to the human medicine clinic, this type of cancer is often detected in advanced stages and the mammography screening technique is not available. The methods to diagnose the stages of the cancer are more difficult to apply and the totality of the examinations is limited to clinical and histological examination, which are given by the surgical intervention on the tumor, and sometimes the regional adenopathology.

In fact, the treatment of the mammary cancer is limited in the majority of the cases to the surgical treatment, which is optimal for the incipient cases, with a less aggressive development. The use of radiotherapy, hormonotherapy, chemotherapy and targeted molecular therapy are limited to isolated cases in the pathology of the canine mammary gland. This is considered to be a paradox, because the mammary cancer in bitches represented and represent a theoretical model to study the mammary cancer in humans and it is a model to validate the efficacy of the chemotherapy (Ciuleanu, 2011).

This situation sustains the need of prescription and a new targeted approach to raise the accessibility to chemotherapy in veterinary domain in general and in pets in special. In scientific veterinary research there is no data concerning the electronic platforms for prescription of the chemotherapy treatment, or for the acceptance of the practitioners to work with an online platform which is updated permanently.

This research aims to test the acceptability regarding the realization and implementation of a multimedia platform to prescribe cytostatic medical treatment in the veterinary medicine, which include relevant information, timely, for taking the therapeutic decision.

\section{MATHERIALS AND METHODS}

We conducted a study based on a survey which was original developed in a focus group with eight veterinarians, in October 2014. The information required concerned the number of items, their content, the utility and acceptability for realization of the program chemotherapy platform for prescription, and also the ethical aspects regarding the limitation of the access of the small animals to chemotherapy prescription due to elevated costs and lack of real time information concerning the optimal prescription.

The survey had 32 items, with pre answered questions, was structured in order to collect data concerning the socio-demographical information of the participants (Q 1-7), the utility ( $Q 10-20$ ) and acceptability of a program to prescribe on the standardized protocols the chemotherapy treatment, the accessibility and utility to work on this type of product, the psychological price of the new online product ( $Q$ 21-24), including also a smart phone application (Q 30-32), ethical aspects regarding treatment in veterinary medicine $(Q$ 25-29). Some of the items in the survey were calibrated as a tool to measure using a Lickert scale with 5 values.

It was tested on a pilot group of 5 participants; their results were excluded from the final results. Collection of the data was realized by selfregistration, face to face, during the January-March 2015 period.

After validation of this survey, it was applied to a batch of people working in the field of veterinary medicine - students of the Faculty of Veterinary Medicine at different educational levels (students and graduate students), veterinary practitioners employed in the public or private veterinary assistance, teachers of the Faculty of Veterinary Medicine. The number of participants in the study was determined based on the formula of Tarro Yaman, with a coefficient error of 0,1 (Mureşan, 1989).

There were criteria developed for the inclusion and exclusion from research of the subjects and all of them expressed their informed consent to participate in the survey anonymously, with the right to use personal information only for the purpose of scientific research and respecting the principles of ethics in scientific research.

Inclusion criteria: student to Veterinary Medicine; veterinary public sector employee; 
authorized veterinarian; veterinarian in private practice; veterinarian committed to the practice (clinic, hospital) private; veterinarian unemployed; retired; Veterinary Medicine graduate and are employed in another field; regardless of age group, gender (female, male) and area of origin (urban, rural); permission to participate in the study.

Exclusion criteria from the study: the refusal of participation in the study.

Data were processed using statistical and mathematical methods. (Muresan, 1989), presenting them being performed in Microsoft Excel.

\section{RESULTS AND DISCUSSION}

Participants in the study belonged to both genders in almost equal percentages (F: 50.94\% and $\mathrm{M}: 49.06 \%$ ), the majority (84.06\%) were aged between 25-59 years, with activities in the field of veterinary medicine, with experience between 1 and 29 years (56.61\%).

Almost half of respondents (48.57\%) says reveals frequently and very frequently in the course of oncological practice.

This distribution suggests that oncological problems may require prescription treatments with cytostatic substances. This supports the need for the concerns regarding a new management approach targeted at increasing accessibility of veterinary oncology treatments in general and for pets in particular.

Data analysis reveals the need felt and expressed by respondents to a multimedia platform for optimizing chemotherapy veterinary prescription medication, claiming that $81.13 \%$ of subjects consider the platform needed and very necessary to have the platform. (Fig. 1)

Over three quarters of respondents - 79.25\% - consider appropriate and very appropriate the format of multimedia platform with applications in veterinary medicine. Data analysis reveals that over two thirds - $67.92 \%$ - of respondents declare that the existence of electronic platform would increase their interest in prescription of chemotherapy medication.

The analysis shows that over half of respondents - $56.60 \%$ - believe that they do not have enough updated information to establish, in good time, the treatment with chemotherapy for patients with oncological pathology in veterinary medicine. The existence of information in printed format (books, articles) requires an additional effort from the practitioner for treatment initiation effort involving longer treatment decision, being a time-consuming activity, less approved by veterinary practitioner in managing the time for practice. (Fig. 2)

Most respondents (94.34\%) say they have easy access to internet communication.

Analyzing the distribution of responses to the question regarding the utility for automatic prescribing software with access to continually updated therapeutic protocols for chemotherapy in veterinary medicine it revealed that over $90.00 \%$ of the respondents declare, to varying degrees (from "less useful" - 7.55\% to "extremely useful" - 30.19\%), the view that the new product would be useful for practical work in veterinary medicine practice.

The effectiveness of information provided in multimedia format by the new proposed product is valued over $95.00 \%$ of respondents. This opinion sustains the need for achievement and implementation of this new multimedia platform, suggesting high acceptability and potential increased addressability of the users for an electronic prescription multimedia program for cancer treatment.

All respondents claim that the introduction of the new product will have an influence on the quality of care for patients with oncological veterinary pathology.

Note that a low share answer that they consider this influence will be very low and low $(9.44 \%)$, but the vast majority $(90.56 \%)$ of responses consider that the influence to quality of care will be great, large and very large.

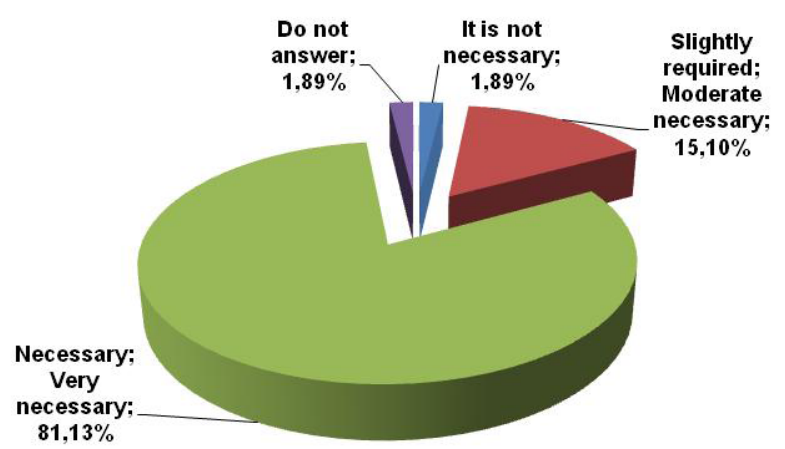

Fig. 1. Proportion of respondents in according to the statement regarding the need of multimedia platform to optimize the prescription of chemotherapy medication in veterinary medicine. 
The analysis reveals a high degree of availability on accessing the electronic source of information in oncology prescription treatment, so just under $4.00 \%$ share of respondents say they are not willing to use electronic program. Note the high percentage of over two thirds of respondents - 69.81\% - which declared themselves ready and very willing to seek information provided by electronic platform for prescription of chemotherapy.

Research has revealed a high degree of utility of the platform (94.34\%), efficiency of information (95.28\%), its positive influence on the quality of care $(90.56 \%)$ and increased availability of access to electronic source (96.23\%).

Analyzing the option to purchase electronic prescribing software for oncology treatment, reveals a high availability. The largest share (94.34\%) of respondents did not rule out the idea of purchasing this new product.

Most respondents (86.79\%) believe that a minimum price without doubting the quality of service offered varies widely between 50 and 400 RON. The maximum price stated they would be willing to pay to purchase electronic product even reaches maximum values in the grid (501-600 RON) (Fig. 3).

This result suggests the importance given by practitioners for veterinary instruments that are easy accessed, with updated information necessary to optimize the quality of care and that are aimed to increase addressability to offered services.

The analysis demonstrates the necessity of the application for the prescription program of cancer therapy accessed from smart phone is considered to be useful by the vast share of respondents 83.02\% (Fig. 4).

For the application to be accessed from a smart phone over three quarters of the respondents $(77.35 \%)$ declare they would be willing to pay a minimum amount between 50 and 200 RON, which sum would not make them question the quality of the product.

Although there is a share of $28.30 \%$ of respondents that consider that they would be willing to pay for a mobile phone application a maximum price between 301 and 600 RON, we can see that the majority - $67.93 \%$ - would prefer that the maximum price for this product does not exceed 300 RON (Fig. 4)

The research was realized based on the original survey that we conceived. We did not use a standard survey instrument because the literature lacks this type of instrument to evaluate the aspects that we were interested for this research. The data collected could not be compared with similar results and can be considered as preliminary results, which need further research on other batches of subjects. This will allow

\section{Without a multimedia platform for optimizing chemotherapy medication prescription in veterinary medicine, do you believe that you have sufficient sources of information, to decide in good time :}

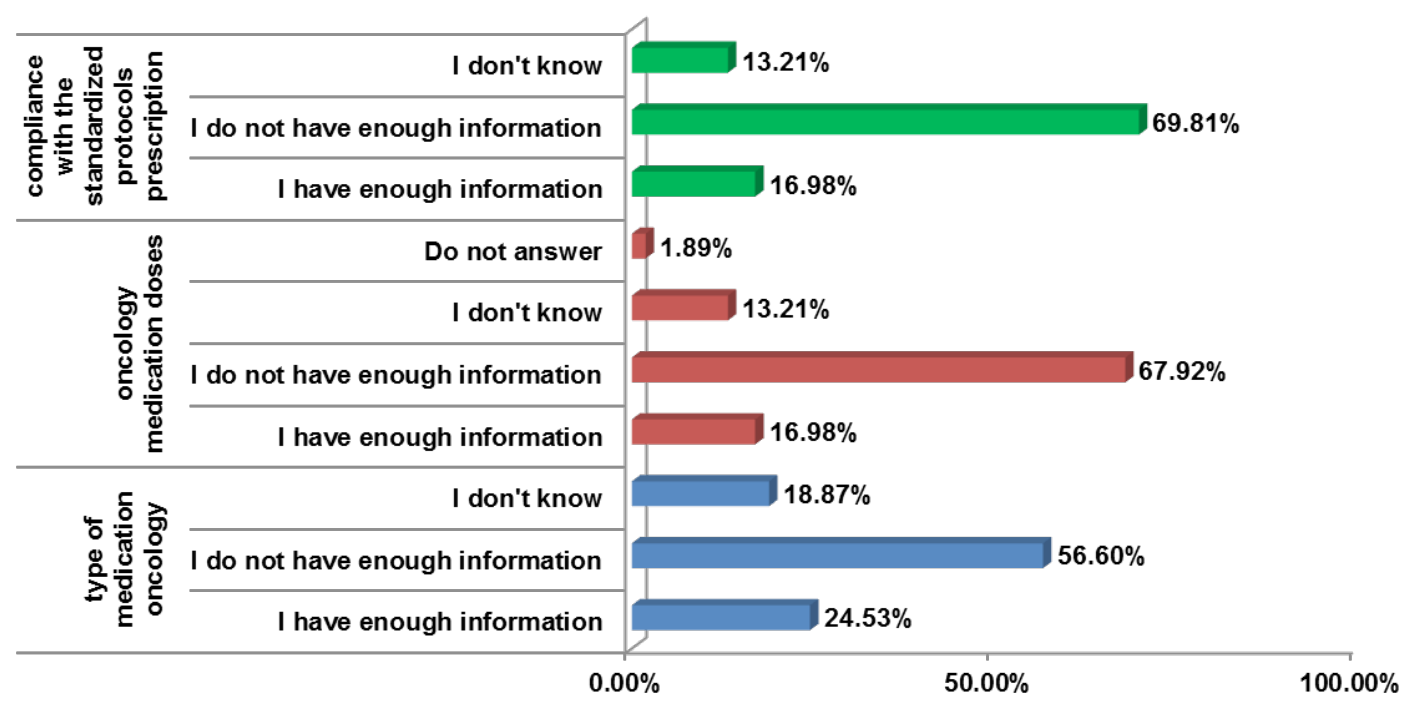

Fig. 2. Share of respondents depending on the limits recognition in decision taking for chemotherapy treatment. 


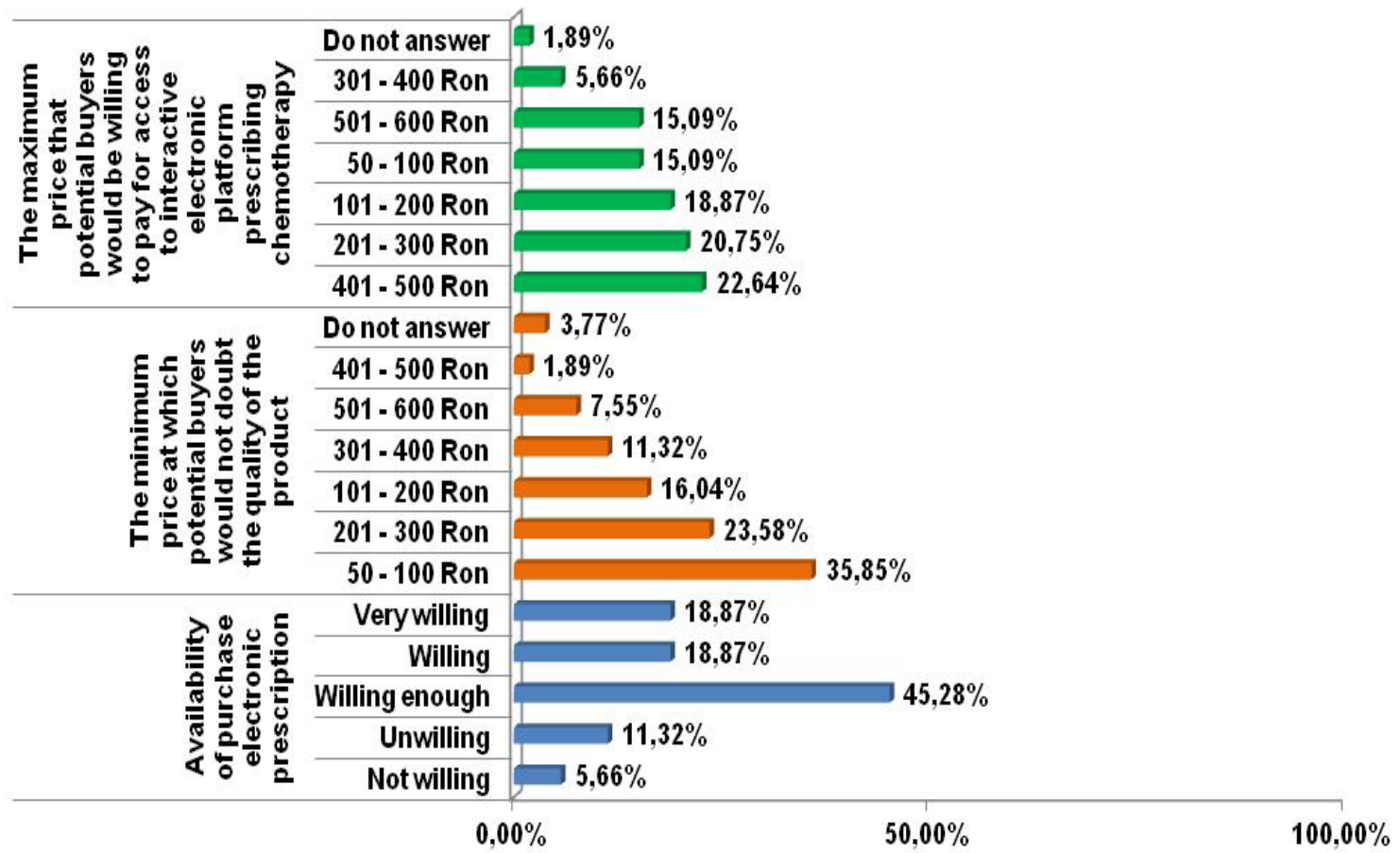

Fig. 3. Share of respondents questioned about the availability of the new product and minimum and maximum price declared acceptable for access to the interactive electronic prescribing chemotherapy platform.

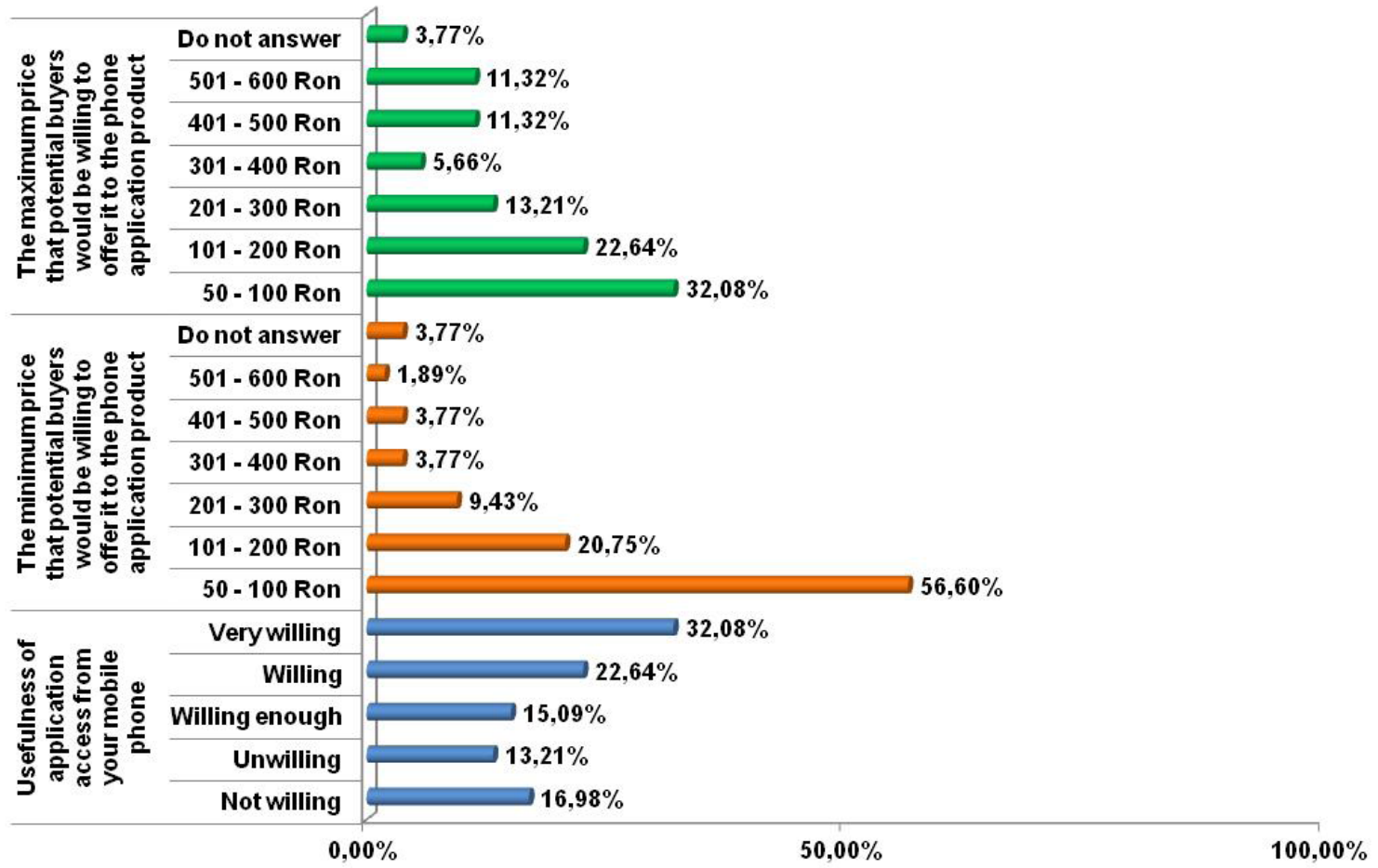

Fig. 4. The share of respondents questioned regarding the utility of the application accessed from a smart phone and the declared acceptable minimum and maximum price for its acquisition. 
comparing the results that we obtained with similar studies. The results cannot be generalized to the whole veterinary community in Romanian; the sample on which the research was made it is not representative for the whole veterinary community.

\section{CONCLUSION}

Data analysis reveals the need felt and expressed by respondents to a multimedia platform for optimizing chemotherapy prescription in veterinary medication, most respondents $(81.13 \%)$ claiming to be necessary and very necessary the existence of the program.

Data analysis reveals that over two thirds of respondents $(67.92 \%)$ declare that the existence of electronic platform would increase interest in chemotherapy prescription medication.

More than half of respondents $(56.60 \%)$ believe they do not have sufficient updated information to establish, in a timely manner, treatment with chemotherapy for patients with oncological pathology in veterinary medicine.

Research has revealed a high degree of utility of the platform (94.34\%), efficiency of information (95.28\%), its positive influence on the quality of care and increased availability of electronic access (96.23\%). and purchasing power for the two types of applications - computer (on line) and mobile phone applications.

The importance given by practitioners for veterinary instruments which are easy accessed, with updated information necessary to optimize the quality of care is suggested by the high availability (94.34\%) of vets to buy the new product: the new multimedia program for veterinary chemotherapy prescription.

Acknowledgements. This paper was published under the frame of European Social Found, Human
Resources Development Operational Programme 2007-2013, Project no. POSDRU/159/1.5/S/ 138776.

\section{REFERENCES}

1. Ciuleanu TE, Todor N (2014). Program de farmacografie clinică în terapia tumorilor solide. Napoca Star, ClujNapoca, 10-86.

2. Ciuleanu LE (2011). Cercetări privind diagnosticul şi terapia tumorilor neoplazice la cățea. Teza licență, USAMV, Cluj-Napoca.

3. Dobson JM, Samuel S, Milstein H et al (2002). Canine neoplasia in the UK estimates of incidence rates from a population of insured dogs. J Small Anim Pract 43:240246.

4. Dorn CR, Taylor DON, Schneider R et al (1968). Survey of animal neoplasms in Alameda and Contra Costa Counties, California. II. Cancer morbidity in dogs and cats from Alameda County. J Natl Cancer Inst 40:307-318.

5. Egenvall A, Bonnett BN, Ohagen P et al (2005). Incidence of and survival after mammary tumors in a population of over 80000 insured female dogs in Sweden from 1995 to 2002. Prev Vet Med 69:109-127.

6. Groza I Șt (2006). Ginecologie, andrologie și obstetrică veterinară - Compendiu. Editura Academiei Române, București, 393-453.

7. Vascellari M, Baioni E, Ru G, Carminato A, Mutinelli F (2009). Animal tumour registry of two provinces in northern Italy: incidence of spontaneous tumours in dogs and cats. http://www.biomedcentral.com/1746$6148 / 5 / 39$.

8. Mureșan P (1989). Manual de metode matematice în analiza stării de sănătate. Editura Medicală, București.

9. Philibert JC, Snyder PW, Glickman N et al (2003). Influence of host factors on survival in dogs with malignant mammary gland tumors. J Vet Intern Med 17:102-106.

10. Reif JS (2007). The Epidemiology and Incidence of cancer. In : Withrow SJ, Vail DM , Withrow \& MacEwen's Small Animal Clinical Oncology, 4th Edition, Saunders Elsevier, chapter 4, 68-76.

11. Withrow SJ, Vail DM, Page Rodney L (2013). Withrow and MacEwen's Small Animal Clinical Oncology, 5th edition, Saunders Elsevier, ISBN 978-1-4377-2362-5. 